\title{
Funcionamiento familiar y resiliencia en estudiantes con facultades sobresalientes del tercer año de secundaria de una institución educativa pública con altas exigencias académicas, durante el período 2013
}

\author{
Family functioning and resilience in students with outstanding powers of the junior year of a public \\ educational institution with high academic standards during the period 2013
}

Teófilo Josué García Pérez' ${ }^{1}$ Zinia Karen Yupanqui Illacutipa1, Katty Puente Guedez²

\begin{abstract}
RESUMEN
Objetivo: Determinar la relación existente entre resiliencia y funcionamiento familiar en una muestra de 231 estudiantes del tercer año del nivel secundario de nueve secciones, en una institución educativa pública con altas exigencias académicas. Material y Métodos: Para evaluar la variable resiliencia se utilizó la Escala de Resiliencia de Young (1993) y para la variable funcionamiento familiar el Cuestionario de Evaluación de Funcionamiento Familiar (CEFF) elaborado por Atri (2006), que está elaborado basado en el Funcionamiento Familiar McMaster (MMFF). Resultados: Los resultados muestran una relación significativa entre las dimensiones de resiliencia y funcionamiento familiar. Se determinó que los estudiantes que provienen de hogares con padres casados califican su familia como funcional y presentan un alto nivel de resiliencia. Conclusiones: Estos resultados sostienen las consideraciones teóricas respecto a determinar el funcionamiento familiar como factor influyente y determinante en la formación de la resiliencia.
\end{abstract}

Palabras clave: Resiliencia, funcionamiento familiar, estudiantes con facultades sobresalientes.

\begin{abstract}
Objective: In this research the relationship between resilience and family functioning is analyzed in a sample of 231 students of the third year of secondary sections of an educational institution publish with high academic standards. Material and Methods: assessing variable resilience resilience Scale was used Young (1993) and for the variable family Functioning Assessment Questionnaire family Functioning (Ceff ) produced by Atri (2006 ) which is developed based on the McMaster model of Family Functioning (MMFF ). Results. The results show a significant relationship between the dimensions of resilience and family functioning. It was determined that students who come from homes with married parents describe their family as functional and have a high level of resilience. Conclusions: This supports the theoretical considerations to consider family functioning as influential and instrumental in the formation of the resilience factor.
\end{abstract}

Keywords: Resilience, family functioning, students with outstanding abilities. 


\section{INTRODUCCIÓN}

Durante los últimos años ha crecido el interés por estudiar a la familia, la cual se considerada el núcleo de nuestra sociedad en donde se prepara a sus miembros, siendo esta un referente social. (Camacho, León y Silva, 2009). Asimismo, Valdés (1997) sostiene que el rol de la familia es muy amplio, pues no se limita tan solo a asegurar la supervivencia física de sus integrantes, sino que además garantiza la integración sociocultural de estos a distintos escenarios donde se desenvuelvan adecuadamente.

Por otro lado, la vida está sujeta a constantes cambios, frente a los cuales es necesario contar con capacidades como la resiliencia la cual se define como la capacidad para resistir, tolerar la presión de los obstáculos y pese a ellos hacer las cosas correctas, bien hechas, cuando todo parece actuar en contra. Puede entenderse, aplicada a la psicología, como la capacidad de una persona de hacer las cosas bien pese a las condiciones de vida adversas, superándolas y saliendo de ellas fortalecido o incluso transformado (Wagnild \& Young (1993).

Según el Ministerio de la Mujer y Poblaciones Vulnerables MIMP (2001, citado por Peña, 2009), el estudio de la resiliencia se da desde un enfoque multidisciplinario, en el que intervienen tanto factores psicológicos (características personales) como socioculturales (familia y comunidad). De este modo, es importante considerar la manera en que las familias intervienen en el desarrollo de la resiliencia, ya que en esta los padres brindan apoyo y dirección afectiva en la vida de sus hijos, además de establecer vínculos importantes que prevalecen a través de largos períodos de tiempo (Conger et al., 2002).

Ponzetti y Long (1989) señalan que un adecuado funcionamiento familiar se caracteriza, básicamente, por la libertad que muestran los integrantes para comunicar clara y directamente sus experiencias y sentimientos, de tal manera que pueden planificar y demostrar capacidad para llegar a acuerdos y resolver los problemas que se manifiestan en la vida cotidiana.

Walsh (1998) describe tres dimensiones que intervienen en la resiliencia y el buen funcionamiento familiar: el sistema de creencias familiares, que permite dar un significado a distintas situaciones y define la manera cómo afrontar problemáticas familiares; los patrones de organización, en los que se comparte el liderazgo, con soporte mutuo y trabajo en equipo; y los procesos de comunicación, donde se dan a entender diferentes puntos de vista, se aclaran ambigüedades, se practica la empatía y se ejercitan estrategias de resolución de problemas.
Por lo descrito anteriormente, se puede ver a la familia como una fuente relevante para desarrollar actitudes que proporcionan a los niños y adolescentes herramientas y recursos para afrontar adversidades, siendo algunas de estas herramientas desarrollar relaciones de confianza y de cuidado, desarrollar un propósito positivo en las expectativas de la vida, sobrellevar decisiones que se han tomado y contar con una familia con cohesión y apoyo. Por lo tanto, se concluye que el ambiente familiar es fundamental para el desarrollo de la resiliencia en los niños (Springer 1997, citado por Athié y Gallegos, 2009).

Por ello, se plantea la siguiente pregunta: ¿Existe relación significativa entre las dimensiones del funcionamiento familiar y resiliencia en estudiantes con facultades sobresalientes del tercer año de secundaria de una institución educativa pública con altas exigencias académicas durante el período 2013 ?

El objetivo de este estudio es determinar si existe relación significativa entre las dimensiones del funcionamiento familiar y resiliencia en estudiantes con facultades sobresalientes del tercer año de secundaria de una institución educativa pública con altas exigencias académicas.

\section{MATERIAL Y MÉTODOS}

La investigación es de diseño no experimental y de corte transversal, porque no se manipularon las variables y porque las datos fueron tomados en un momento dado. El alcance es descriptivo correlacional, ya que se describe la resiliencia y el funcionamiento familiar y la relación existente entre ellos. (Hernández, Fernández y Baptista. 2004).

\section{Participantes}

Los estudiantes evaluados fueron 231 de sexo masculino y sexo femenino, matriculados en el tercer año de secundaria del CMSPP, de las nueve secciones.

\section{Instrumentos}

Cuestionario de Evaluación del Funcionamiento familiar (EFF).

Para la evaluación del funcionamiento familiar, se aplicó el Cuestionario de Evaluación de Funcionamiento familiar (CEFF) elaborado por Atri en 1987, el cual fue validado y publicado en el año 1993, siendo copilado posteriormente por Velazco y Luna, 2006. El presente cuestionario está basado en el Modelo de Funcionamiento Familiar McMaster (McMaster Model of Family Functioning - MMFF). 
Funcionamiento familiar y resiliencia en estudiantes con facultades sobresalientes del tercer año de secundaria de una institución educativa pública con altas exigencias académicas, durante el período 2013

Fue validado y estandarizado con reactivos elaborados de acuerdo a un lenguaje accesible a la comprensión de población de estudio. Este cuestionario es del tipo Likert y utiliza cinco opciones para valorar cada reactivo: totalmente de acuerdo (5), de acuerdo (4), Ni de acuerdo ni en desacuerdo (3), en desacuerdo (2) y totalmente en desacuerdo (1).

Los 6 factores que presenta el Cuestionario de Evaluación del Funcionamiento Familiar son: involucramiento afectivo funcional, involucramiento afectivo disfuncional, patrones de comunicación funcional, patrones de comunicación disfuncional, resolución de problemas y patrones de control de conducta. El CEFF se validó a juicio de expertos y docentes de la Universidad Peruana Unión en el año 2011, la fiabilidad se obtuvo a través del alfa de Cronbach, donde el índice de confiabilidad fue de 0 . 610.

\section{Escala de Resiliencia de Wagnild y Young}

La Escala de resiliencia fue construida por Wagnild y Young en 1988, y fue revisada por los mismos autores en 1993. Está compuesta de 25 ítemes, los cuales puntúan en una escala tipo Likert de 7 puntos, donde 1 es en desacuerdo, y un máximo de acuerdo es 7 . Los participantes indicarán el grado conformidad con el ítem, ya que todos los ítemes son calificados positivamente, los más altos puntajes serán indicadores de mayor resiliencia, el rango de puntaje varía entre 25 y 175 puntos.

La Escala de Resiliencia tiene como componentes: confianza en sí mismo, ecuanimidad, perseverancia, satisfacción personal, sentirse bien solo. El CEFF se validó a juicio de expertos y docentes de la Universidad Peruana Unión en el año 2011. Y según el Alfa de Cronbach, el índice de confiabilidad de la escala es de 0.850 .

\section{RESULTADOS}

Tabla 1

Niveles de funcionamiento familiar en estudiantes con facultades sobresalientes del tercer año de secundaria de una institución educativa pública con altas exigencias académicas, durante el período 2013.

\begin{tabular}{|c|c|c|c|c|c|c|}
\hline \multirow[t]{3}{*}{ Dimensiones del funcionamiento familiar } & \multicolumn{6}{|c|}{ Nivel } \\
\hline & \multicolumn{2}{|c|}{ Bajo } & \multicolumn{2}{|c|}{ Medio } & \multicolumn{2}{|c|}{ Alto } \\
\hline & $\mathrm{F}$ & $\%$ & $\mathrm{~F}$ & $\%$ & $\mathrm{~F}$ & $\%$ \\
\hline Involucramiento afectivo funcional & 64 & 28 & 104 & 45 & 63 & 27 \\
\hline Involucramiento afectivo disfuncional & 58 & 25 & 109 & 47,3 & 64 & 27,7 \\
\hline Patrones de comunicación funcionales & 38 & 16,4 & 160 & 69,3 & 33 & 14,3 \\
\hline Patrones de comunicación disfuncionales & 59 & 25,5 & 111 & 48 & 61 & 26,5 \\
\hline Resolución de problemas & 67 & 29 & 131 & 56,7 & 33 & 14,3 \\
\hline Patrones de control, de conducta & 53 & 23 & 113 & 49 & 65 & 28 \\
\hline
\end{tabular}

En la tabla 1 se observa que, en las dimensiones del funcionamiento familiar, la mayoría de los estudiantes se encuentran en un nivel medio, presentando los siguientes porcentajes: involucramiento afectivo funcional $=45 \%$, involucramiento afectivo disfun- cional $=47,3 \%$, patrones de comunicación funcionales $=69,3 \%$, patrones de comunicación disfuncionales $=48 \%$, resolución de problemas $=56,7 \%$ y patrones de control de conducta $=49 \%$. 
Tabla 2

Niveles de resiliencia en estudiantes con facultades sobresalientes del tercer año de secundaria de una institución educativa pública con altas exigencias académicas, durante el periodo 2013.

\begin{tabular}{|c|c|c|c|c|c|c|}
\hline \multirow{3}{*}{ Dimensiones de Resiliencia } & \multicolumn{6}{|c|}{ Nivel } \\
\hline & \multicolumn{2}{|c|}{ Bajo } & \multicolumn{2}{|c|}{ Medio } & \multicolumn{2}{|c|}{ Alto } \\
\hline & $\mathrm{F}$ & $\%$ & $\mathrm{~F}$ & $\%$ & $\mathrm{~F}$ & $\%$ \\
\hline Confianza en sí mismo & 66 & 28,4 & 99 & 43 & 66 & 28,6 \\
\hline Ecuanimidad & 68 & 29,4 & 110 & 47,6 & 53 & 23 \\
\hline Perseverancia & 60 & 26 & 107 & 46,3 & 64 & 27,7 \\
\hline Satisfacción personal & 67 & 29 & 122 & 53 & 42 & 18 \\
\hline Sentirse bien solo & 64 & 28 & 120 & 52 & 47 & 20 \\
\hline Resiliencia total & 68 & 29,4 & 97 & 42 & 66 & 28,6 \\
\hline
\end{tabular}

En la tabla 2 se da a conocer que los estudiantes, en su mayoría, presentan un nivel medio de Resiliencia total (42\%). Asimismo, se encuentran cada una de las dimensiones: confianza en sí mismo (43\%), ecuanimidad $(47,6 \%)$, perseverancia $(46,3 \%)$, satisfacción personal (53\%), sentirse bien solo (52\%).

Tabla 3

Análisis de correlación de Pearson entre funcionamiento familiar y resiliencia en estudiantes con facultades sobresalientes del tercer año de secundaria de una institución educativa pública con altas exigencias académicas, durante el período 2013.

\begin{tabular}{llll}
\hline Dimensiones de la CEFF & \multicolumn{2}{l}{ Correlación de Pearson } \\
& $\mathrm{R}$ & Sig. & Decisión \\
\hline Involucramiento afectivo funcional &, $343^{* *}$ &, 000 & Significativo \\
Involucramiento afectivo disfuncional &,$- 379^{* *}$ &, 000 & Significativo \\
Patrones de comunicación funcionales &, $415^{* *}$ &, 000 & Significativo \\
Patrones de comunicación disfuncionales &,$- 196^{* *}$ &, 003 & Significativo \\
Resolución de problemas &, $326^{* *}$ &, 000 & Significativo \\
Patrones de control, de conducta &, 018 &, 790 & No significativo \\
\hline
\end{tabular}

En la tabla 3 se muestra que existe relación significativa entre las dimensiones: de funcionamiento familiar y resiliencia: involucramiento afectivo funcional y resiliencia $(\mathrm{r}=, 343 \mathrm{p}=0,000)$, involucramiento afectivo disfuncional y resiliencia $(\mathrm{r}=-, 347$, $\mathrm{p}=, 000)$, patrones de comunicación disfuncionales $\mathrm{y}$ resiliencia $(\mathrm{r}=-196, \mathrm{p}=, 003)$, Patrones de comunica-

\section{DISCUSIÓN}

La superación y el éxito que demuestra una persona a pesar de haber afrontado adversidades, siendo considerada como resiliente, permite el surgimiento de esta investigación que busca conocer si existe relación significativa entre la resiliencia y el funcionamiento familiar, en adolescentes talentosos, ción funcionales y resiliencia $(r=, 415, \mathrm{p}=, 000)$, resolución de problemas y resiliencia $(r=, 326, p=, 000)$ funcionamiento familiar general y resiliencia $(r=, 406$, $\mathrm{p}=, 000)$. Sin embrago, a diferencia de las dimensiones anteriores, se observa que no existe significativa relación entre patrones de control, de conducta y resiliencia $(\mathrm{r}=, 018, \mathrm{p}=, 000)$. 
familiar y la resiliencia en estudiantes con facultades sobresalientes del tercer año de secundaria de una institución educativa pública con altas exigencias académicas durante el año 2013, esto significa que cuanto más alto sea el nivel de funcionamiento familiar, mayor será el nivel de resiliencia, esto corrobora lo que dice Valdés (1997), quien sostiene que el rol de la familia va más allá de asegurar la supervivencia física, es decir, no solo aporta beneficios físicos, sino que, además, proporciona los recursos necesarios para la integración sociocultural de sus miembros a escenarios donde puedan desenvolverse, afrontar y superar adecuadamente las adversidades. Asimismo, estudios realizados por Ponzetti y Long (1989), en jóvenes universitarios, han demostrado que aquellos estudiantes que reciben apoyo de sus familias obtienen un promedio académico superior, determinando así que la familia influye en la formación y desarrollo académico, además, dicha relación positiva en el contexto familiar no solo influye en el nivel de educación básico, sino también en el nivel superior. Conjuntamente, Athié y Gallegos (2009), en su investigación realizada entre funcionamiento familiar y resiliencia en poblaciones que se enfrentan a situaciones de adversidad, sostienen que sí existe relación significativa entre estas variables y sus respectivos factores.

Seguidamente, se obtiene como resultado que el involucramiento, afectivo funcional se relaciona significativamente y de manera directa, con la resiliencia $(r=, 343 \mathrm{p}<0.000)$, lo cual corrobora el resultado obtenido respecto al segundo objetivo específico, el cual señala que existe una relación significativa inversa entre el involucramiento afectivo disfuncional y el nivel de resiliencia $(\mathrm{r}=$ ,$- 379 \mathrm{p}<0.000$ ), esto da a entender que al presentar un mayor nivel de involucramiento afectivo, mayor será el nivel la resiliencia del individuo, por lo tanto, a menor nivel de funcionamiento familiar menor será el nivel de resiliencia. Tal y como lo sostiene Atri (2006), al mencionar que el involucramiento afectivo es el grado en que la familia muestra interés, valora a cada miembro de la familia y denota la habilidad para responder con una amplia gama de emociones, así como la capacidad de proporcionar cuidados, afecto y bienestar, por lo tanto, la investigación realizada muestra que estas son algunas de las habilidades que una persona necesita para desarrollar resiliencia. Además Palomar y Márquez (1999) sostienen que algunas de las contribuciones que da la familia a la resiliencia del individuo son: relaciones de confianza y de cuidado, el desarrollo de un propósito positivo en las expectativas de la vida, soportar decisiones que se han tomado y proveer una familia con cohesión y apoyo.
Adicionalmente, se aprecia que existe relación significativa directa entre los patrones de comunicación funcionales y el nivel de resiliencia $(\mathrm{r}=, 415 \mathrm{p}<0.000)$, de la misma manera existe relación significativa inversa entre los patrones de comunicación disfuncionales y el nivel de resiliencia $(r=-, 196 p<0.003)$. Esto concuerda con lo que mencionan Ponzetti y Long (1989), que una característica principal del funcionamiento familiar, es la expresión clara y directa de los integrantes, de tal manera que se da un diálogo abierto, llegando a resolver conflictos que se suscitan en la vida cotidiana. De este modo, Atri (2006) refiere que los patrones de comunicación tienen que ver con el intercambio de información verbal, principalmente en el área instrumental.

Posteriormente se encuentra que la resolución de problemas se relaciona significativamente, y de manera directa, con el nivel de resiliencia $(r=, 326 \mathrm{p}<$ 0.000 ), esto da a entender que al presentar un mayor nivel de resolución de problemas, mayor será el nivel la resiliencia. Lo cual reafirma lo que menciona la Organización Panamericana de la Salud (1997), que la habilidad para la resolución de problemas es una característica de los adolescentes con un nivel alto de resiliencia. Dicha afirmación concuerda con lo señalado por Atri (2006), quien menciona que las familias, con una adecuada organización, tienen la capacidad de resolver problemas que se presentan en el diario vivir.

Por otro lado, a diferencia de los resultados obtenidos anteriormente, se encontró que los patrones de control de conducta no se relacionan significativamente con el nivel de resiliencia, $(r=$ ,018 $\mathrm{p}<0.790)$, contrarrestando lo mencionado por Atri (2006), quien define los patrones de control de conducta como aquellos modelos que adopta una familia para manejar el comportamiento; caracterizado por la flexibilidad y la tolerancia, los cuales son considerados por Dallos (1996, citado por Villalba, 2004), como elementos básicos de la resiliencia.

La relación ausente entre los patrones de control de conducta y la resiliencia señala que las normas que adopta una familia, respecto al comportamiento de sus miembros, no influyen sobre el nivel de resiliencia de sus integrantes. Esto significa que el nivel de resiliencia no depende de lo flexible o inflexible y de lo tolerante o intolerante que sean las normas de comportamiento en familia del estudiante.

Finalmente, de manera general, los resultados de esta investigación corroboran que existe una 
relación significativa entre las dimensiones del funcionamiento familiar y la resiliencia. Lo que nos permite elaborar programas que aumenten el nivel de resiliencia, mediante la mejora del funcionamiento familiar.

Declaración de financiamiento y de conflicto de intereses:

El estudio fue financiado por la autora, quien declara no tener algún tipo de conflicto de interés en la investigación realizada.

\section{Correspondencia}

Teófilo Josué García Pérez

Universidad Peruana Unión, Carretera Central Km. 19.5 Ñaña.

Correo electrónico:josuegape@hotmail.com

\section{REFERENCIAS BIBLIOGRÁFICAS}

Ahié, D. y Gallegos, P. (2009). Relación entre resiliencia y el funcionamiento familiar. Revistas Científicas de América Latina. 1 (17), 5 -14. Recuperado de http://redalyc.uaemex.mx/src/inicio/ArtPdfRed. jsp? iCve $=133912613002$

Atri, 2006. Cuestionario de Evaluación del Funcionamiento Familiar (EFF). Velasco, M., Luna, M., (Eds.). Instrumentos de evaluación en terapia familiar y de pareja, (,1-27.). México: Paxmexico.

Camacho, P., León, C. y Silva, M. (2009). Funcionamiento familiar según el modelo Circumplejo de Olson en adolescentes. Herediana, 2 (2), 80-85. Recuperado de http://www.upch.edu.pe/faenf/images/stories/ articulorevista2_2/olson_2_2_5

Conger, R., Wallace, L., Sun, Y., Simons, R., McLoyd, V. y Brody, G. (2002). Economic Pressure in African American Families: A Replication and Extension of the Family Stress Model. Developmental Psychology, $38(2), 179-193$.
Hernández, R., Fernández, C. y Baptista P. (2004). Metodología de la investigación. 3. ${ }^{\mathrm{a}}$ edición. México: McGraw-Hill-Interamericana.

Peña, N. (2009). Fuentes de resiliencia en estudiantes de Lima y Arequipa. Revista Liberabit. 15(1): 59-64. Recuperado de http://pepsic.bvsalud.org/pdf/liberabit/ v15n1/v15n1a07.pdf

Ponzetti, J. y Long, E. (1989). Healthy Family Functioning: a Review and Critique. Family Therapy, 16 (1), 43-50.

Walsh, F. (1998). Strengthering Family Resilience. Nueva York: Guilford Press.

Recibido: 09/05/2014 Aceptado: 02/11/2014 